\title{
Characterization studies on the novel mixed thin films
}

\author{
P. Ramasundari ${ }^{1}$, S. Ganeshan ${ }^{2}$ and R. Vijayalakshmi ${ }^{3}$ \\ ${ }^{1}$ P. G. \& Research Department of Physics, S. V. N. College, Madurai, India \\ ${ }^{2}$ Department of Physics, Vivekananda College, Madurai, India \\ ${ }^{3}$ P. G. \& Research Department of Physics, Thiagarajar. College, Madurai, India
}

ramprof62@gmail.com, ramyarv@rediffmail.com

\section{PACS 68}

DOI $10.17586 / 2220-8054-2016-7-4-683-686$

\begin{abstract}
Among electrochemical processes having a considerable impact on technical development, the mixed thin film (Mo-Ni oxide) plays an important role, due to its better mechanical, anticorrosive and thermal stability characteristics. The mixed films have been prepared by dip spin coating. The films are grown on substrates like Indium Tin Oxide (ITO) and are well adherent on the substrates, pinhole free and transparent. The X-ray diffraction analysis of the films confirms they are polycrystalline in nature. The morphological study reveals that the uniform distributions have flower-like structure. From the compositional analysis, the EDAX spectra show the presence of molybdenum and nickel. The optical band gap was found to be $1.36 \mathrm{eV}$ and band assignments for Fourier Transform Infrared (FTIR) spectra are comparable to reported values.
\end{abstract}

Keywords: Dip Spin Coating, EDAX, SEM, X-Ray Diffraction.

Received: 5 February 2016

Revised: 27 April 2016

\section{Introduction}

The optical properties of thin films are very important for many applications. Researchers have shown considerable interest in Ni-Mo oxide films, because of their applications such as hydrogen evolution catalysis [1,2] and anticorrosion treatment [3]. Transition metal oxides, like nickel oxides have found application due to their anti-ferromagnetic semiconducting properties with a wide band gap of $3.6 \mathrm{eV}$ [4,5]. This provides opportunities for use in electrochromic devices [6], fuel cells [7] or other applications. Electrodeposition-prepared transition metal oxide films exhibit good electrochromic properties. For electrodeposited Mo-Ni oxide films, it has been shown that as Mo content increases, certain peaks in XRD broaden and disappear due to a decrease in grain size [8,9]. Even though there are reports for the optical properties, structural characteristics, band assignments and electrical properties by dip spin coating Indium Tin Oxide (ITO) plates, values are reported only for Indium Tin Oxide (ITO) substrates in this paper.

\section{Experimental}

The concentrations of $0.05 \mathrm{~N}$ (of $\mathrm{MoO}_{3}$ and $\mathrm{NiSO}_{4}$ mixture) at $330 \mathrm{~K}$ by dip spin coating under optimized conditions. The coating was performed at $3000 \mathrm{rpm}$ for $30 \mathrm{mins}$ as optimized parameters to obtain the mixed oxide thin film. The coated samples were investigated by conventional XRD with $\mathrm{CuK} \alpha$ radiation using XPERT-PRO diffractometer. FTIR measurements in the range of $400-4000 \mathrm{~cm}^{-1}$ were carried out by JASCO CANVAS. The SEM photographs were recorded using HITACHI S-3400.

\section{Results and Discussion}

\subsection{XRD Analysis}

The structural properties of these films were analyzed by the X-ray diffractometer. Fig. 1 shows the diffraction pattern of Mo-Ni mixed oxide thin film which is deposited on the Indium Tin Oxide (ITO) plate. From the surface analysis, it was found that the mixed thin films exhibit uniform thickness. The diffracting angles were set to $20^{\circ}$ to $80^{\circ}$ with a low scanning speed and in continuous mode. Diffraction peaks were observed at $2 \theta=28.450$, $39.430,44.720,47.320$ and 65.560, corresponding to the $\left(\begin{array}{lll}0 & 0 & 2\end{array}\right),\left(\begin{array}{lll}-2 & -2 & 2\end{array}\right),\left(\begin{array}{lll}1 & 2 & 2\end{array}\right),\left(\begin{array}{lll}1 & -1 & 3\end{array}\right),\left(\begin{array}{lll}-1 & -5 & 1\end{array}\right)$ planes of the mixed thin films respectively. XRD pattern of Mo-Ni mixed oxide thin films showed polycrystalline wurzite structure with a preferential $\left(\begin{array}{lll}1 & 2 & 2\end{array}\right)$ orientation peak and the peaks are comparable to reported values [10]. 


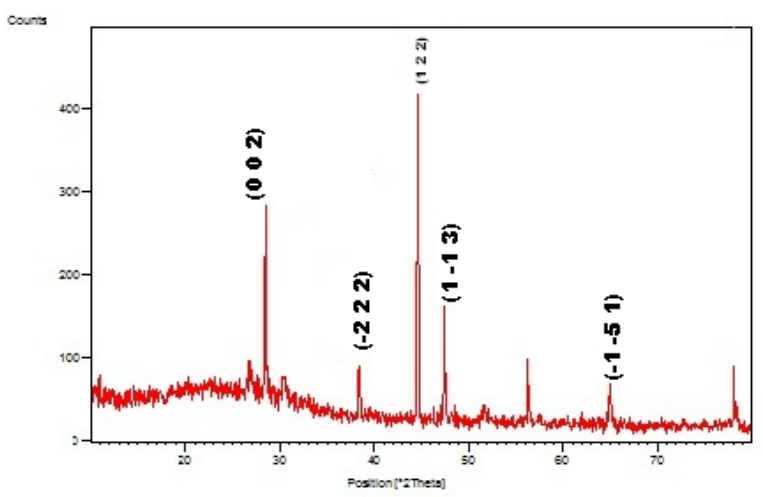

FIG. 1. XRD pattern of as deposited Mo-Ni oxide film on ITO substrate

\subsection{SEM Analysis}

The film deposited on the Indium Tin Oxide (ITO) substrate showed flower-like structure. The EDAX, which was used in conjunction with SEM, confirmed the elemental composition of the film as deposited on the substrate, revealing the presence of molybdenum and nickel $(89.52 \% \mathrm{Mo} \& 5.42 \% \mathrm{Ni})$ as well as impurities like copper and zinc.
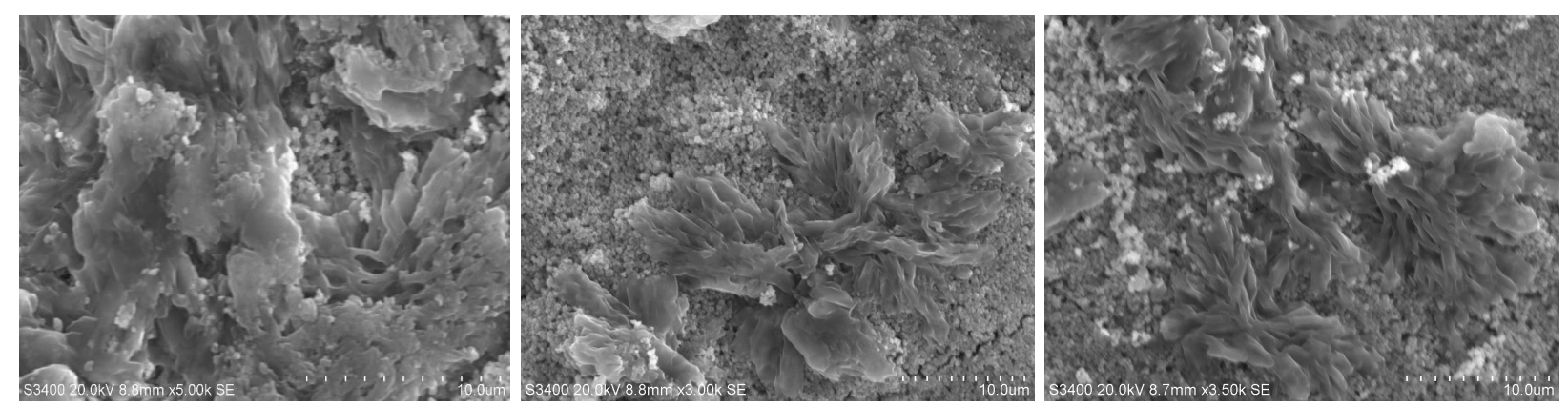

FIG. 2. SEM images of as deposited Mo-Ni oxide film on ITO substrate

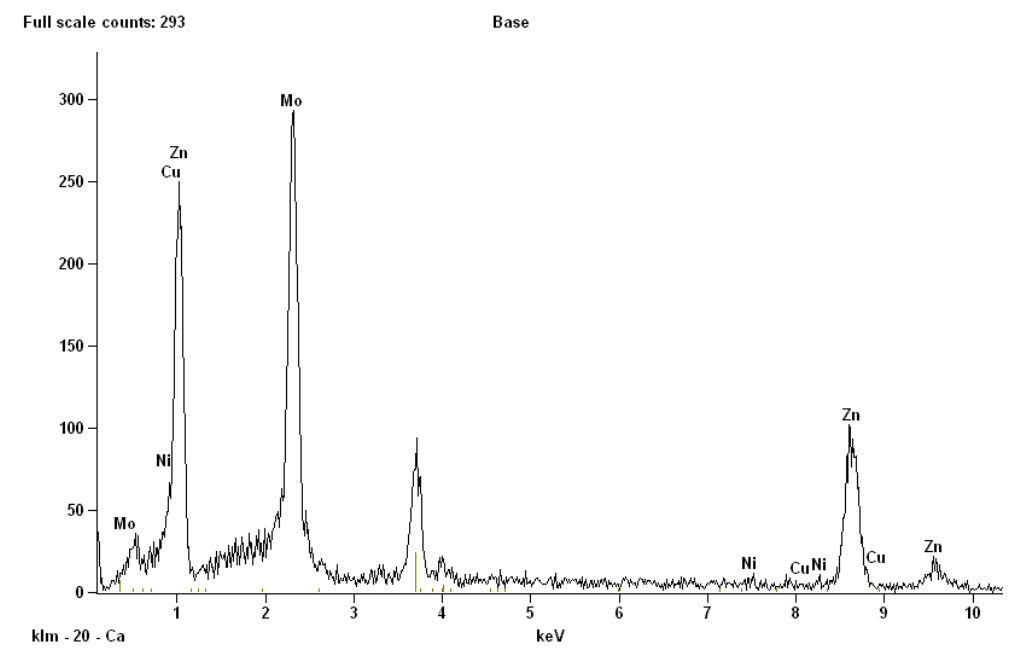

FIG. 3. EDAX spectra of as deposited mixed Mo-Ni oxide film on ITO substrate 


\subsection{FTIR Spectra}

Fourier transform infrared spectroscopy provides information about a particular compound's functional groups, molecular geometry and inter/intramolecular interactions. The bands at $3425 \mathrm{~cm}^{-1}, 1614 \mathrm{~cm}^{-1}$ in Fig. 4 were attributed to the surface absorbed water in the mixed Mo-Ni oxide thin films. The band $3780 \mathrm{~cm}^{-1}$ and $3932 \mathrm{~cm}^{-1}$ are due to $\mathrm{O}-\mathrm{H}$ stretching vibrations of water molecules. The vibration absorption peaks at $875 \mathrm{~cm}^{-1}$ were due to Mo-O-Mo stretching [11].

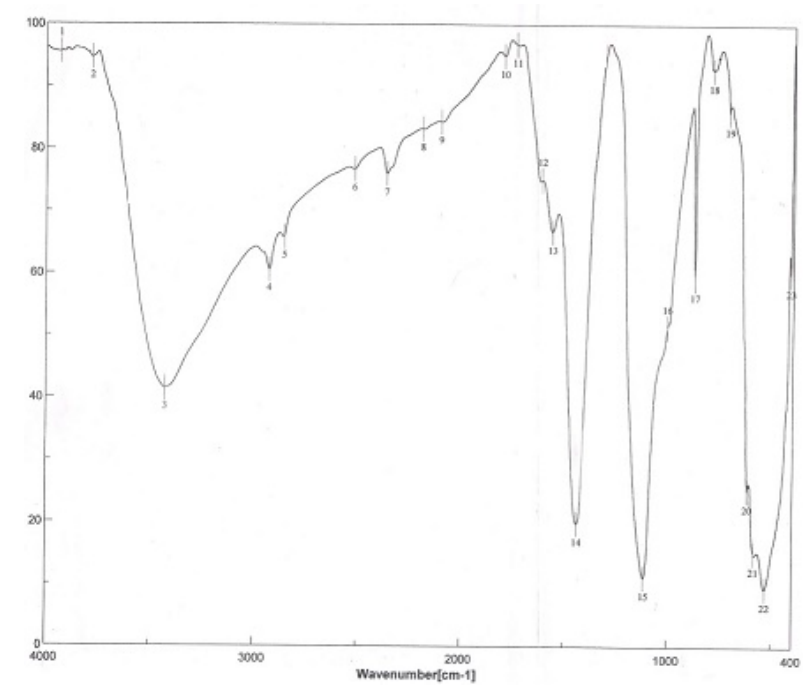

FIG. 4. FTIR Spectra of as deposited mixed Mo-Ni oxide film

\subsection{Optical Studies}

Optical absorption characterisitcs were studied in the $200-1100 \mathrm{~nm}$ range. The absorption spectrum for the as-deposited mixed Mo-Ni oxide film is shown in Fig. 5. The UV-Visible spectrum shows a sharp peak centered at $360-376 \mathrm{~nm}$ with a long tail towards longer wavelength side. Absorption has very high value in the UV region and decreases with increasing wavelength, becoming constant towards the visible region, which indicates a high transparency in the visible region. The optical band gap of mixed Mo-Ni thin film was found to be $1.36 \mathrm{eV}$.

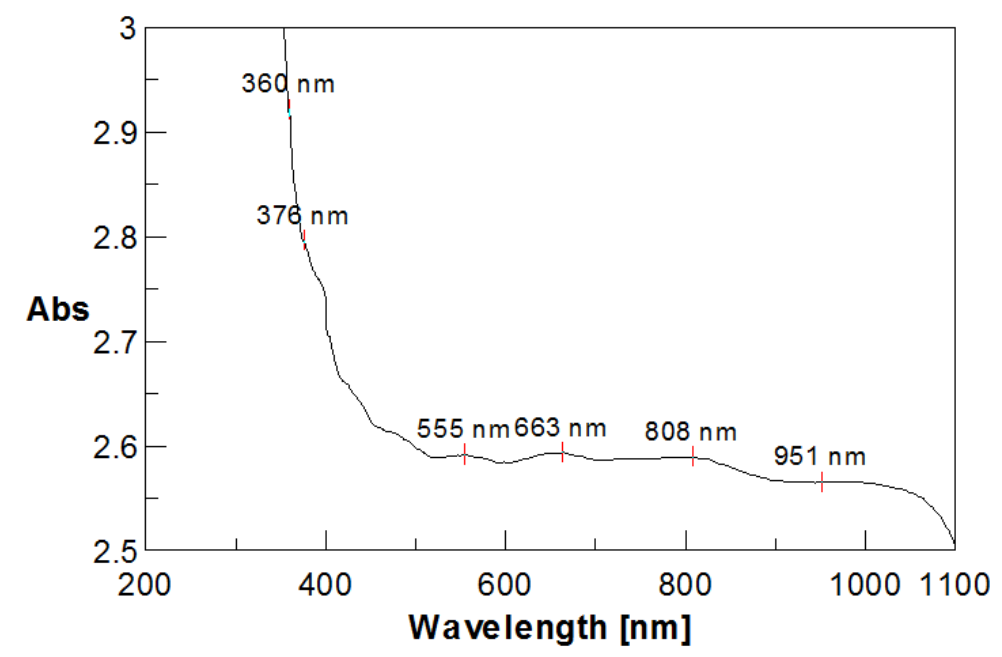

FIG. 5. Absorprtion Spectra for as deposited mixed Mo-Ni oxide film on ITO substrate 


\section{Conculsion}

Electrochromic materials, like mixed Mo-Ni oxide thin film, were deposited on ITO under optimized conditions. The dip spin coated films are white in color and are transparent. The films are well adherent on the substrates, pinhole-free and transparent. X-ray diffraction shows that the films are polycrystalline in nature and are comparable to JCPDS data. SEM images reveal that the films are without any pinholes or cracks and cover the substrate well. The optical absorption spectrum reveals that the material has a direct band gap nature and its energy is in agreement with the reported values.

\section{References}

[1] Mihailov L., Spassov T., Kanazirski I., Tsvetnaov I. Electrocatalytic behavior of Ni-based amorphous alloys for hydrogen evolution. J. Mater. Sci., 2011, 46, P. 7068-7073.

[2] Kirk D.W., Thorpe S.J., Suzuki H. Ni-base amorphous alloys as electrocatylst for alkaline water electrolysis. Int. J. Hydrogen Energy, 1997, 22, P. 493-500

[3] Chassaing E.E., Portail N.N., Levy A.F., Wang G. Electrodeposition and Characterization of Nanocrystalline Ni-Mo Catalysts for Hydrogen Production. J. Appl. Electrochem., 2004, 34, P. 1085-1091.

[4] Adler D., Fainleb J.J. Electrical and optical properties of 'Narrow?Band Materials'. Phys. Rev. B, 1970, 2 (8), P. $3112-3134$.

[5] Zollner M., Kipp S., Becker K.D. Reactive Processes of Nickel Oxide on oxidic Substrates as observed by Scanning Force Microscopy. Crystal Research and Technology, 2000, 35 (3), P. 299-305.

[6] Purushothaman K.K., Muralidharan G. Nanostructured NiO Based All Solidstate electrochromicdevice. Journal of Sol-Gel Science and Technology, 2008, 46, P. 190-197.

[7] Shaigan N., Ivey D.G., Chen W. Metal?Oxide Scale Interfacial Imperfections and Performance of Stainless Steels Utilised as interconnects in Solid Oxide Fuel cells. Journal of The Electrochemical Society, 2009, 156 (6), P. 765-770.

[8] Beltowaka-Lehman E., Bigos A., Indykaand P. Electrodeposition and characterisation of nanocrystalline Ni-Mo coatings. Surf. Coat. Technol., 2012, 211, P. 67-71.

[9] Beltowaka-Lehman E., Indyka P.P. Kinetics of Ni-Mo electrodepsotion from Ni-rich citrate baths. Thin Solid Films, $2012, \mathbf{5 2 0}$, P. 2046.

[10] Masatoshi Saitou. Characterization of Electrodeposited Ni and Ni?Mo Thin Films by X-ray Diffraction. Int. J. Electrochem. Sci., 2014, 9, P. 6033-6042.

[11] Sulma Marisela Fernandez-Valverde, Eduardo Ordonez-reqil, Gerardo cabanas-Moreno, Omar Solorza-feria. Electrochemical Behavior of Ni-Mo Electrocatalyst for Water Electrolysis. J. Mex. Chem. Soc., 2010, 54 (3), P. 169-174. 